\title{
AUTOMATIC CONTROL OF A LOCATING DEVICE DETECTING AND TRACKING AN AIR TARGET
}

\author{
K. Stefański*, L. Nocoń ${ }^{* *}$
}

\begin{abstract}
The paper presents the optical system suspended on the Cardan joint, performing space scanning to detect and track aerial infrared emitting targets. The essence of the paper is the use of a special device control algorithm. This algorithm consists in using the phase trajectories of control deviations. It is possible to achieve a scanning time of 1 second at a target moving at transonic speed. The scanning range can achieve \pm 60 [deg] in the horizontal and vertical plane. Numerical simulations were carried out using the Matlab software, and the results of some of the tests were presented in a graphical form.
\end{abstract}

Keywords: control, scanning, tracking, air target, phase trajectories

\section{Introduction}

The seeking for a target in space can be done directly by a shooter equipped with a daytime or night vision telescopic sight. In more advanced devices, the process of seeking for a target takes place automatically. It is then an automatic space search or a space scanning. After locating the target, the scanning system should determine its position, and then start its tracking. It can be assumed that the target is depicted by an illuminated point or a small surface emitting infrared radiation.

By means of optoelectronic devices, the position of the target is most often determined using two angles: pitch and yaw from the optical axis of the device. In general, the distance of the target from the focal length of the device is not determined.

The method discussed here was taken from work Hsu, 1968 and used to control the axis of the scanning and tracking device. It is based on determining the phase trajectories of control deviations. However, the device itself is a scanning and tracking head consisting of a gyroscope and optical, mechanical and electronic elements and subassemblies that allow the implementation of a set task (Dziopa, 2015 and Gapiński et al., 2014) made of materials such as metals, composites and plastics. However, the paper does not discuss the detailed construction of the device - only its automatics is considered.

\section{Implementation of the device}

This device belongs to the passive devices, using target's own radiation in the range of infrared. The automation of the device's operation has been divided into two ranges: 1) space scanning and target locating; 2) tracking of infrared radiation emitted by the target. The optical system is fixed in the rotor axis of the gyroscope with three degrees of freedom suspended on the Cardan joint in such a way that the optical axis coincides with the axis $\eta$ of the rotor (Fig. 1).

Space scanning is accomplished by controlling the motion of the gyroscope axis, which draws a developing conical surface.

\footnotetext{
Assistant Prof. Konrad Stefański, Ph.D. Eng.: Faculty of Mechatronics and Mechanical Engineering, Kielce University of Technology, al. Tysiąclecia P.P. 7; 25-314, Kielce; PL, kstefanski@tu.kielce.pl

** Assistant Prof . Łukasz Nocoń, Ph.D. Eng.: Faculty of Mechatronics and Mechanical Engineering, Kielce University of Technology, al. Tysiąclecia P.P. 7; 25-314, Kielce; PL, lnocon@tu.kielce.pl
} 


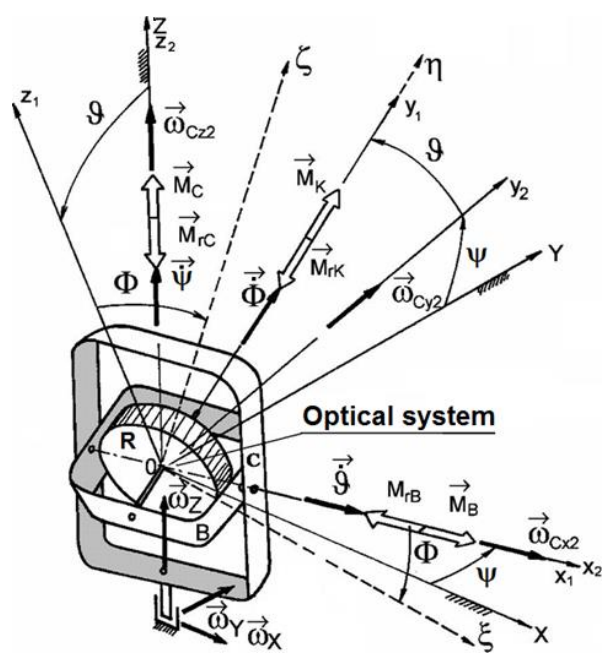

Fig. 1: The diagram of a gyroscope suspended on the Cardan joint

The control signals (desirable) cause that the optical axis determines, on a located at some distance spherical surface concentric with the $\eta$ axis, the curve dependent on the algorithm: Archimedean spiral or rosette.

\section{Equations and results of numerical simulations}

Equations of motion of the gyroscope axis were assumed in the nonlinear range, for any value of pitch and yaw angles, with linear characteristics of resistance forces (viscous) in Cardan joint bearings (Gapiński, 2017). They were derived by means of Lagrange's equations of the second kind and they are as follows:

$$
\begin{aligned}
& M_{B}=J \ddot{\vartheta}+M_{r B}+J \dot{\psi}^{2} \sin \vartheta \cos \vartheta-J_{0} n \dot{\psi} \cos \vartheta \\
& M_{C}=J \cos ^{2} \vartheta \ddot{\psi}+M_{r C}-J \dot{\vartheta} \dot{\psi} \sin 2 \vartheta+J_{0} n \dot{\vartheta} \cos \vartheta
\end{aligned}
$$

where: $J, J_{0}\left[\mathrm{kgm}^{2}\right]-$ moments of inertia of gyroscope rotor (with optical system) $\mathrm{n}$ relation to its transversal and longitudinal axis; $M_{r B}=b b \dot{\vartheta}$ i $M_{r C}=b c \dot{\psi}$ [Nm] - moments of damping forces in inner and outer gyroscope frames bearings; $M_{B}, M_{C}[\mathrm{Nm}]$ - moments of forces acting on the inner and outer frame of gyroscope; $n[\mathrm{rad} / \mathrm{s}]$ - rotational speed of the rotor.

From the equation (1) one indicated:

$$
\begin{gathered}
u=\ddot{\vartheta}=\frac{M_{B}-b b \dot{\vartheta}-J \dot{\psi}^{2} \sin \vartheta \cos \vartheta+J_{0} n \dot{\psi} \cos \vartheta}{J} \\
v=\ddot{\psi}=\frac{M_{C}-b c \dot{\psi}+J \dot{\vartheta} \dot{\psi} \sin 2 \vartheta-J_{0} n \dot{\vartheta} \cos \vartheta}{J \cos ^{2} \vartheta}
\end{gathered}
$$

Control algorithms have the following form (Hsu, 1968 and Osiecki, 2003):

$$
\begin{gathered}
p=-u \operatorname{sgn}\left(0.5\left|e_{1}\right| e_{1}+u e_{0}\right) \\
r=-v \operatorname{sgn}\left(0.5\left|e_{3}\right| e_{3}+v e_{2}\right)
\end{gathered}
$$

The control of the gyroscope axis motion was realized by means of a trajectory of control deviations going to zero. The diagram of this control is shown in Fig. 2. At the moment of transition from the scanning state to the tracking state, in the block defining the control signals $p$ and $r$ one should introduce new deviations: in place of $e_{0}$ substitute $e_{u}=\vartheta-\varepsilon_{t}$; in place of $e_{1}$ substitute $e_{u 1}=\dot{\vartheta}-\dot{\varepsilon}_{t}$; in place of $e_{2}$ substitute $e_{v}=\psi-\sigma_{t}$; in place of $e_{3}$ substitute $e_{v 1}=\dot{\psi}-\dot{\sigma}_{t}$. The values $\varepsilon_{t}(t)$ and $\sigma_{t}(t)$ are the angular coordinates of the moving target. Figures 3 and 4 show results for two examples of numerical simulations. 


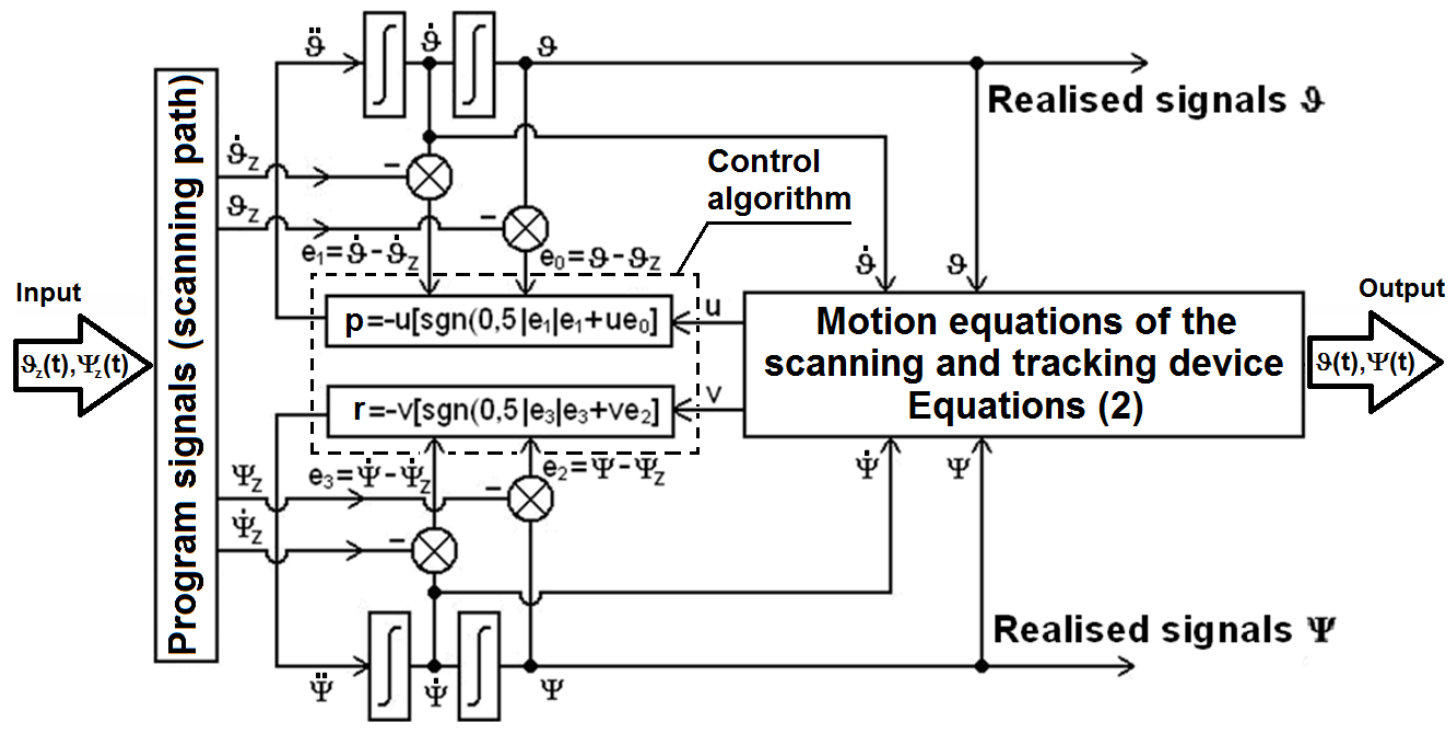

Fig. 2: Block diagram of scanning and tracing a target

\subsection{Numerical simulation - example 1}

The program control of the device's axis motion was carried out according to the Archimedean spiral on the equations:

$$
\vartheta_{z}=a t \sin \omega t ; \quad \psi_{z}=a t \cos \omega t
$$

where: $a=0.6[\mathrm{rad}] ; \omega=50.27[\mathrm{rad} / \mathrm{s}] ; t-$ time.

The initial target location amounted to: $\varepsilon_{t 0}=0.2[\mathrm{rad}], \sigma_{t 0}=0.75[\mathrm{rad}]$; initial distance of the target from the optical system: $D=2000[\mathrm{~m}]$; target velocity: $V=300[\mathrm{~m} / \mathrm{s}]$. The flight path of the target was determined by the following equations:

$$
\varepsilon_{t}=\varepsilon_{t 0}+\frac{V}{D} t^{2} ; \quad \sigma_{t}=\sigma_{t 0}+\frac{V}{D} t
$$

The calculations were carried out according to the diagram shown in Fig. 2 for the following parameters of the gyroscope: $J=0.0032\left[\mathrm{kgm}^{2}\right] ; J_{0}=0.0076\left[\mathrm{kgm}^{2}\right] ; b b=b c=0.05[\mathrm{Nm} / \mathrm{s}] ; n=600[\mathrm{rad} / \mathrm{s}]$.

The final tracing error was determined by the equation:

$$
e_{k}=\sqrt{\left(\vartheta-\varepsilon_{t}\right)^{2}+\left(\psi-\sigma_{t}\right)^{2}}
$$

This error has reached the value of: $e_{k}=1.68 \cdot 10^{-3}[\mathrm{rad}]$. The interception of the target took place after time: $t_{t}=0.523[\mathrm{~s}]$.

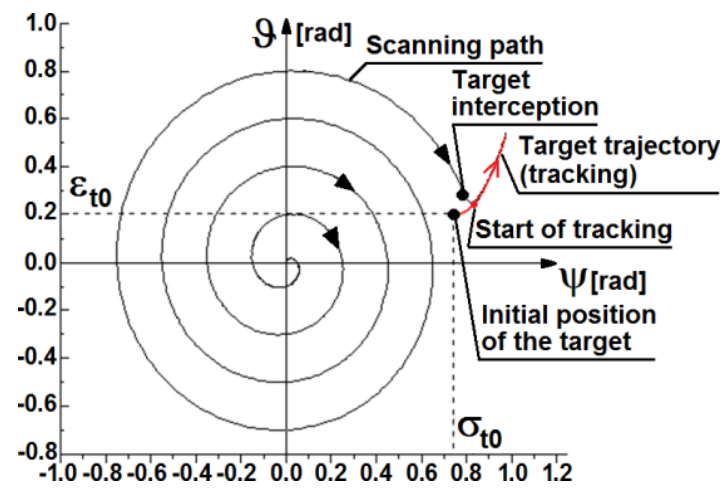

Fig. 3: Schematic representation of digital simulation-example one

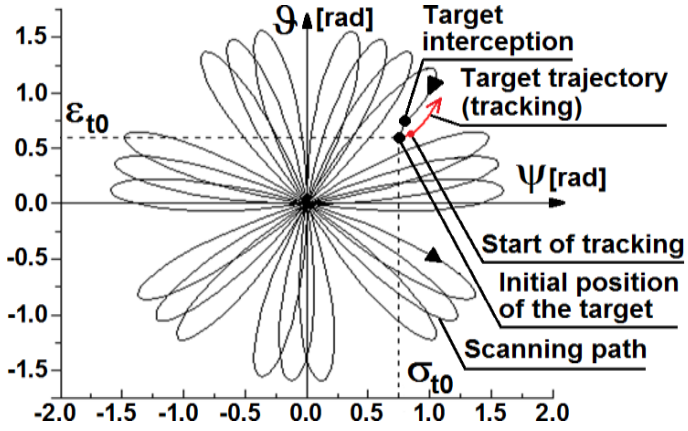

Fig. 4: Schematic representation of digital simulation - example two 


\subsection{Numerical simulation - example 2}

The program control of the device's axis motion was carried out according to the rosette on the equations:

$$
\vartheta_{z}=a \sin \omega_{1} t \sin \omega t ; \quad \psi_{z}=a \sin \omega_{1} t \cos \omega t
$$

where: $a=1.6[\mathrm{rad}], \omega=50.27[\mathrm{rad} / \mathrm{s}], \omega_{1}=6.67 \omega[\mathrm{rad} / \mathrm{s}]$.

The initial target location amounted to: $\varepsilon_{t 0}=0.75[\mathrm{rad}], \sigma_{t 0}=0.6[\mathrm{rad}]$; initial distance of the target from the optical system: $D=1000[\mathrm{~m}]$; target velocity $V=300[\mathrm{~m} / \mathrm{s}]$. Flight path of the target was determined by the equations (5). The calculations were carried out according to the diagram shown in Fig. 2 for the parameters of the gyroscope identical as in example 1. The final tracking error is defined by the equation (6) : $e_{t}=3.05 \cdot 10^{-4}[\mathrm{rad}]$. The interception of the target took place after time $t_{t}=0.204[\mathrm{~s}]$.

These examples show the high effectiveness of the presented target scanning and tracking system. The dimensions of the gyroscope are reasonable: the diameter of the rotor $d=0.06[\mathrm{~m}]$, mass of the rotor $m=0.55[\mathrm{~kg}]$. Short target interception time and minor tracking errors should be noticed. The applied control method, based on phase trajectories of errors, works faster and more accurately than the PID controller (Szmidt et al., 2017 and Osiecki, 2003). The limitations can be the coordinate values of the initial position of the target and its velocity. The interception of the target should take place in such a position that it is not able to go beyond the scanning zone before it is captured.

\section{Conclusions}

The analyzed system, consisting of a gyroscope with three degrees of freedom, in the axis of which an optical system receiving signals from the target in the infrared range is placed, allows the use of various scanning paths after reception of the first signals emitted by the target from a wide area in space. It directs the optical axis to the highest value of the radiation emitted by the target, regardless of the energy distribution of this radiation. It is effective even when the target changes its position at high velocity. After locating the target, the device switches to tracking the path of its flight.

Thus, the presented system complies with the formulated task. Control of the gyroscope axis is uncomplicated, the scanning time until the target interception is short, and the tracking is characterized by high accuracy. It is possible to mount the device in the target coordinator, e.g. in air-air missiles. Then, the pilot would not have to intercept the target directly by maneuvering the plane, and the system will work just like in the missile "Python-4". The device can also be used in coordinators of other homing missiles, for example, ground-to-air type such as "Grom" or newer "Piorun".

\section{References}

Dziopa, Z. and Koruba, Z. (2015) The impact of launcher turret vibrations control on the rocket launch. Bulletin of the Polish Academy of Sciences - Technical Sciences, Vol. 63, No. 3, pp. 717-728.

Gapiński, D. and Szmidt, P. (2017) The control process of a scanning and tracking IR seeker using inverse dynamics, in: Engineering Mechanics 2017, Brno University of Technology, Brno, pp. 326-329. Gapiński, D., Krzysztofik, I. and Koruba, Z. (2014) Analysis of the dynamics and control of the modified optical target seeker used in anti-aircraft rocket missiles. Journal of Theoretical and Applied Mechanics, 52, 3, pp. 629-639.

Hsu, J.C. and Meyer, A.U. (1968) Modern control principles and application. McGraw-Hill, New York.

Osiecki, J.W. and Stefański, K. (2003) Controlling of the course and bearing indicator on the basis of phasis trajectories (in polish). Automation and exploitation of control and communication systems, Vol. 2, pp. 421-428.

Szmidt, P., Gapiński, D. and Koruba, Z. (2017) The analysis of selection optimal parameters of PID controllers for a modified artillery-missile system, in: Engineering Mechanics 2017, Brno University of Technology, Brno, pp. 970-973. 